COMMUNICATION

\title{
Comparative Study of Laser Spray and Electrospray Using an Orthogonal Time-of-Flight Mass Spectrometer
}

\author{
Atsushi Takamizawa, ${ }^{\text {a) }}$ Susumu Fujimaki, ${ }^{\text {a) }}$ \\ Shigemitsu OKAZAKI, ${ }^{\text {a) }}$ and Kenzo HiRAoKA, ${ }^{* a)}$
}

(Received February 3, 2004; Accepted February 25, 2004)

\begin{abstract}
A preliminary study on the laser spray and electrospray was made using an orthogonal time-of-flight mass spectrometer. Explosive vaporization and mist formation occurred when an aqueous solution effusing out from the tip of the stainless steel capillary was irradiated from the opposite side of the capillary by a $10.6 \mu \mathrm{m}$ infrared laser. Little ion signals could be detected when the plume was sampled through the ion sampling orifice. When a high voltage $(\sim 3 \mathrm{kV})$ was applied to the stainless steel capillary, strong ion signals appeared. The ion intensities were found to be more than one order of magnitude stronger than those obtained by the conventional electrospray for aqueous solutions of arginine and lysozyme. The present method is regarded as the electric-field assisted MALDI in which the LC solvents (water etc.) act as liquid matrices. The laser spray will be a versatile method for the biological mass spectrometry because this method is compatible with the natural solvent, water.
\end{abstract}

\section{Introduction}

The ultimate goal for the analysis of ions in liquid or solid samples is to achieve rapid evaporation of the liquid samples or solid matrices, to isolate positive and negative ions generated in the gas phase and to transport all of them to the mass spectrometer. In electrospray, the formed charged liquid droplets suffer from the Rayleigh fission accompanied by the evaporation of the solvent resulting in the charge enrichment in the off-spring droplets. ${ }^{1)}$ Some part of the excess charges will ultimately become gaseous ions by the ion evaporation and/or the charged residue mechanisms. ${ }^{1)}$ It is known that the efficiency of the positive and negative ion separation in the Taylor cone becomes less than 1 for the ion concentrations of $>\sim 10^{-5} \mathrm{M}^{1}{ }^{1)}$ In order to increase the sensitivity for electrospray, nanoelectrospray (nanoES) was developed. ${ }^{2}$ In fact, finer charged liquid droplets were formed by nanoES. Because the size of the electrosprayed charged droplets is controlled by the balance between the surface tension and the electrostatic force, it would be difficult to scale down the size of the charged droplets to the atomic level by the spontaneously formed Taylor cone due to the surface tension of the liquid, i.e., the size formed by the nanoES may be closer to the minimum attainable by the electrostatic liquid atomization method. For example, the aqueous electrolyte solution (water has a high surface tension) with a concentration of $>\sim 10^{-3} \mathrm{M}$ is intrinsically difficult to handle. It would be necessary to rely on another auxiliary techniques such as nebulizer, laser irradiation, etc. in addition to high electric field to form the smaller charged droplets or ultimate molecular ions.

Matrix-assisted laser desorption ionization (MALDI)

*a) Clean Energy Research Center, University of Yamanashi (Takeda-4, Kofu 400-8511, Japan) is a possible alternative for the biological mass spectrometry. It relies on the ultraviolet or infrared laser irradiation on the liquid (e.g., glycerol) or solid matrices. After the laser irradiation there is a time delay of tens to hundreds of nanoseconds for the start of the ion desorption. ${ }^{3)}$ In the process of ablation (or spallation) of the condensed matrix sample, various phenomena take place in the plume such as ion-molecule reactions (proton transfer reactions, charge exchange reactions, ion-molecule clustering reactions, etc.), recombination reactions of positive and negative ions, unimolecular dissociations of the sample and matrix molecules, etc. Therefore, there is a possibility for further modifications of the intact samples. In addition, enormous ejections of tiny droplets occur due to the phase explosion followed by recoil-induced expulsion of a large mass of matrix. ${ }^{3), 4)}$ This leads to a serious loss of the sample prepared, i.e., the sacrifice of the sensitivity for the sample detection. If suppression of the liquid droplet formation and complete vaporization of the matrix were realized, tremendous improvement of the detection sensitivity could be achieved.

The pioneering work on the vaporization of liquid using a $10.6 \mu \mathrm{m}$ infrared laser was done by Vestal et $a l{ }^{5)}$ This new approach for a liquid chromatograph/ mass spectrometer (LC/MS) interface used laser vaporization of the LC effluent at atmospheric pressure. The mist and vapor formed were sampled into the vacuum as a molecular beam and were ionized by chemical ionization (CI) or electron ionization (EI). The experiments succeeded in measuring EI mass spectra of a number of compounds injected in liquid solution at flow-rates up to $1 \mathrm{~mL} / \mathrm{min}$ for a number of common solvents such as methanol, acetonitrile, hexane, and chloroform. However, the maximum flow-rate of aqueous solutions that were able to vaporize in a stable manner was about $0.5 \mathrm{~mL} / \mathrm{min}$. They encountered problems with mist formation due to incomplete va- 
porization.

Brutschy et al. studied the laser induced liquid beam ionization/desorption mass spectrometry. ${ }^{6}$ ) This method allows ions to be desorbed directly from the liquid beam injected into the vacuum chamber through a 10 $\mu \mathrm{m}$ nozzle and can be analyzed using a time-of-flight mass spectrometer. They detected the intact hemoglobin using this method for the first time. In this method, a greater part of liquid sample was carried away to the cold trap at liquid nitrogen temperature.

In this paper, preliminary results for the laser spray obtained by using an orthogonal time-of-flight mass spectrometer will be given. It was found that laser spray has a better sensitivity than electrospray by more than one order of magnitude. Since the solvent in the LC effluent acts as a matrix, the laser spray may be regarded as the electric-field assisted MALDI.

\section{Experimental}

A stainless steel capillary (inner diameter: $0.13 \mathrm{~mm}$, outer diameter: $0.3 \mathrm{~mm}$ ) parallel to the interface plate is supplied with a sample solution. Nebulizer gas, $\mathrm{N}_{2}$, effusing from the concentric stainless steel tube (inner diameter: $0.7 \mathrm{~mm}$ ) reduces the angular divergence of the plume and entrains the mist and gas in a confined gas stream going towards the sampling orifice.

A SYNRAD (Bothel, WA, USA), model FSV20SFB, 20 $\mathrm{W}$ infrared laser $(10.6 \mu \mathrm{m})$ is used. The tip of the stainless steel capillary is irradiated axially from the opposite side of the capillary by a laser beam focused to $\approx 0.3 \mathrm{~mm}$. The ions formed at atmospheric pressure are sampled into the vacuum system through a $400 \mu \mathrm{m}$ diameter orifice and mass analyzed by an orthogonal time-of-flight mass spectrometer (Accu-TOF, JEOL, Musashino, Tokyo) over the range of $1-10,000$. The flow rate of the sample solution is $3-100 \mu \mathrm{L} / \mathrm{min}$. The smaller flow rate of the sample solution $(<3 \mu \mathrm{L} / \mathrm{min})$ may also be possible by using the reduced laser power, e.g., the application of the laser spray to nanoESI. Water solvent was prepared by purification of the distilled water with ion exchange resins.

\section{Results and Discussion}

Although an explosive vaporization of the liquid sample took place by the laser irradiation, little ions could be detected without applying high voltage to the stainless steel capillary. This result is similar to that obtained by the atmospheric pressure MALDI, in which no ions could be detected when the water (or ice) sample on the substrate was irradiated by a UV or an IR laser. ${ }^{7-9)}$

Since the present mass spectrometer system was originally designed for electrospray, it was possible to compare the sensitivities of electrospray with that of laser spray. Figure 1 displays the mass spectra for an aqueous solution of $10^{-5} \mathrm{M}$ arginine with laser off (a) and laser on (b). The voltage applied to the capillary was $2,800 \mathrm{~V}$. All the experimental conditions for (a) and (b) were exactly the same except for the laser on and off. Thus the effect of the laser irradiation on electrospray could be studied by this kind of operation. In this experiment, no buffer such as $\mathrm{CH}_{3} \mathrm{COONH}_{4}$ was added to the sample solution. In addition, no special

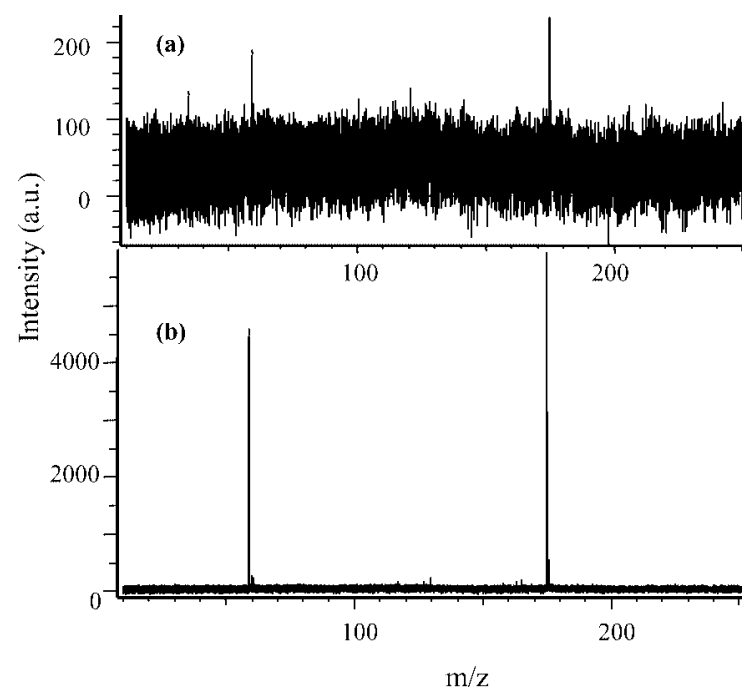

Fig. 1. Mass spectra for an aqueous solution of $10^{-5} \mathrm{M}$ arginine with laser off (a) and laser on (b) (laser power: $8 \mathrm{~W}$ ) obtained by using the orthogonal time-of-flight mass spectrometer (AccuTOF, JEOL). Flow rate of the sample solution: $50 \mu \mathrm{L} /$ min. Flow rate of the nebulizer gas $\mathrm{N}_{2}$ : $0.5 \mathrm{~L} /$ min. The voltage applied to the capillary: 2,800 $\mathrm{V}$. The ion with $m / z 59$ originates from the impurity acetone.

(a)

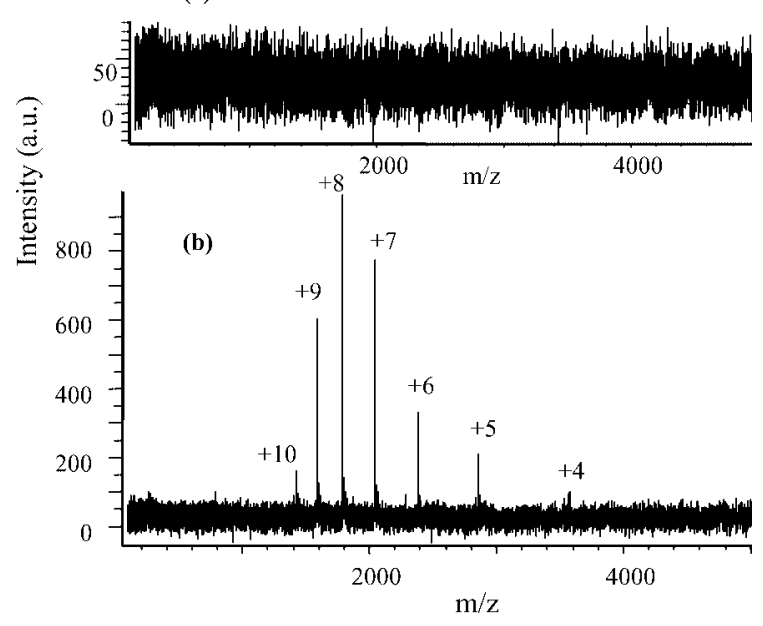

Fig. 2. Mass spectra for an aqueous solution of $10^{-5} \mathrm{M}$ lysozyme chloride with laser off (a) and laser on (b) (laser power: $2 \mathrm{~W}$ ) obtained by using the orthogonal time-of-flight mass spectrometer (AccuTOF, JEOL). Flow rate of the sample solution: $10 \mu \mathrm{L} / \mathrm{min}$. Flow rate of the nebulizer gas $\mathrm{N}_{2}: 0.5 \mathrm{~L} / \mathrm{min}$.

optimization was made for obtaining the stronger ion signals for both laser spray and electrospray because the main objective of the present work was to compare the relative sensitivities for laser spray and electrospray. With the concentration of $10^{-5} \mathrm{M}$ arginine, the ion signal of arginine just started to be observed by the electrospray under the present experimental conditions. With laser on, the intensity of protonated arginine signal $(m / z 175)$ increased by a factor of about 40.

Figure 2 shows the mass spectra for an aqueous solution of $10^{-5} \mathrm{M}$ lysozyme chloride with laser off (a) 
and laser on (b). While lisozyme ions could hardly be observed by electrospray, fairly strong multiplycharged lysozyme ions could be detected by the laser irradiation. These experimental results clearly indicate that the laser spray is superior to the conventional electrospray. The superiority of laser spray to electrospray becomes more evident with larger flow rates of the sample solutions. It was found that the detection limit for lysozyme in aqueous solution by laser spray was at least one order of magnitude lower than that by electrospray with the smallest flow rates of a few $\mu \mathrm{L} /$ min measured in the present experiment.

The time constant for acoustic energy dissipation, $\tau_{\mathrm{ac}}$, is defined by the ratio of laser penetration depth and velocity of sound in the matrix. For aqueous solution $\tau_{\mathrm{ac}}$ may be estimated to be $\sim 10^{-8} \mathrm{~s}$. Hatanaka et al. studied nanosecond and femtosecond laser photochemistry and ablation dynamics of neat liquid toluene. ${ }^{3)}$ They found that the start of the formation of shock wave takes about 22 ns for liquid toluene after the irradiation of the femtosecond laser $\left(\mathrm{Ti}^{3+}: \mathrm{Al}_{2} \mathrm{O}_{3} / \mathrm{KrF}\right.$ hybrid laser, $248 \mathrm{~nm}, 300-500 \mathrm{fs}$ ) at a laser fluence of $90 \mathrm{~mJ} / \mathrm{cm}^{2}$. This value roughly corresponds to $\tau_{\mathrm{ac}}=$ $\sim 10^{-8} \mathrm{~s}$ for the aqueous solution. We measured the minimum time required for the explosive vaporization of the liquid water to be $\sim 0.7 \mu$ s for $50 \mathrm{~W}$ infrared laser with a laser spot of $0.1 \mathrm{~mm}$ diameter (irradiance: $\sim 10^{6}$ $\left.\mathrm{W} / \mathrm{cm}^{2}\right)^{10}{ }^{10}$ This is also compatible with the start of spallation of the liquid toluene at about 580 ns measured by Hatanaka et al. ${ }^{3)}$ They found that the liquid spallation lasts as long as a few ms or even longer after the femtosecond laser pulse. This is one of the reasons why the resolution of the TOF mass spectra for MALDI is poor when the delayed extraction of desorbed ions is not adopted. Another finding made by Hatanaka et al. . $^{3)}$ is that a greater part of the liquid toluene was ablated as tiny liquid droplets, i.e., the efficient vaporization of the liquid sample by the laser irradiation is inherently difficult. This is related to the fact that only a small fraction of sample molecules mixed in the matrices is detected as gaseous ions in MALDI. In this respect, Karas et al. proposed the "lucky-survivor model". ${ }^{11)}$ Overall, these results demonstrate that the desorption of sample ions mixed in matrices from the condensed phase to the gas phase and their isolation for the mass spectrometric detection are very inefficient processes.

By the application of voltage $(V)$ on the metal capillary, the strong electric field $(E)$ is exerted on the tip of the capillary,

$$
E=2 V /[r \ln (4 d / r)]
$$

where $r$ is the radius of the capillary and $d$ is the distance between the capillary and the counter electrode. The value of $E$ was about $10^{5} \mathrm{~V} / \mathrm{cm}$ under the present experimental conditions. The liquid effusing out of the stainless steel capillary experiences this high electric field and the positive ions (in the positive ion mode) are likely concentrated near the meniscus of the liquid and negative ions diffuse to the opposite direction and are neutralized on the metal surface (vice versa in the negative ion mode). The positive ions enriched near the surface of the liquid meniscus will be selectively desorbed by the laser irradiation resulting in high efficiency for the ion extraction. Hale and
Querry ${ }^{12)}$ measured the absorption coefficient of water for $10.6 \mu \mathrm{m}$ infrared light to be $\alpha=832 \mathrm{~cm}^{-1}$. This value leads to the absorption thickness in bulk water $(1 / \alpha)$ to be $\sim 12 \mu \mathrm{m}$. That is, the penetration depth of the laser light in the aqueous solution is at most $\sim 12$ $\mu \mathrm{m}$ thick. The thickness of the electric double layer depends on the electric field strength and the electrolyte concentration. If the electric double layer is thicker than $\sim 12 \mu \mathrm{m}$, an efficient desorption of enriched ions near the liquid surface would be realized. For electrospray, the Taylor cone is formed spontaneously on the tip of the metal capillary due to the high electric field exerted on the tip of the metal capillary. Since the average size of the electrosprayed charged droplets is about a few $\mathrm{mm}$, the curvature diameter of the tip of the Taylor cone may have about the same size. From the Gauss' law and the number of excess charges in the electrosprayed charged droplets ${ }^{1)}$, the electric field on the tip of the Taylor cone can be estimated to be more than one order of magnitude higher than that generated on the tip of the metal capillary $\left(\sim 10^{5} \mathrm{~V} / \mathrm{cm}\right)$. This high electric field on the tip of the Taylor cone (i.e., thick electric double layer) is related to the formation of highly charged droplets. For laser spray, however, the stable formation of Taylor cone may be disturbed by the explosive vaporization of the liquid by the laser irradiation. In other words, the electric field strength generated on the liquid surface for laser spray may be weaker than that on the tip of the spontaneously formed Taylor cone (i.e., the electric double layer for laser spray may be thinner than that for electrospray). The fact that the laser spray has the better sensitivity than the electrospray suggests that the laser spray can form finer liquid droplets than the electrospray, in accordance with the experimental observation.

\section{Conclusion}

Many useful LC/MS interface and soft ionization methods have been developed so far. They rely on nebulizer, electric field, and/or energy sudden techniques. ${ }^{12)}$ The present laser spray is a hybrid of all these. The high electric field exerted on the liquid effusing out of the metal capillary enriches the positive (or negative) ions on the surface of the liquid. The thin layer of liquid which is enriched by the positive (or negative) ions is selectively desorbed by the laser irradiation. The evaporated solvent acts as a nebulizer because the jet flow would lead to further atomization/ vaporization of the liquid droplets entrained in the jet stream, e.g., destruction and evaporation of liquid droplets at the boundary of the Mach disc. The angle of the laser spray cone is found to be much wider than that of the electrospray, indicating higher efficiency for the positive (or negative) ion extraction from the liquid solution. Consequently, these effects result in better sensitivity than electrospray. As described above, the laser spray may be regarded as the electric-field assisted MALDI and it is well suited for the analysis of biological samples because it can readily be applied to aqueous solutions which are relatively difficult to handle by electrospray. The laser spray will be a versatile interface for coupling of mass spectrometer and the on-line liquid separation technique. 


\section{Ackonwledgment}

This work was supported by a Grant-in-Aid from the Japanese Ministry of Education, Science and Culture.

\section{References}

1) P. Kebarle and L. Tang, Anal. Chem., 65, 972A (1993).

2) M. Wilm and M. Mann, Anal. Chem., 68, 1 (1996).

3) K. Hatanaka, Y. Tsuboi, H. Fukumura, and H. Masuhara, J. Phys. Chem. B, 106, 3049 (2002).

4) A. Vogel and V. Venugopalan, Chem. Rev., 103, 577 (2003).

5) C. R. Blakley, M. J. McAdams, and M. Vestal, J. Chromatogr., 158, 261 (1978).

6) A. Wattenberg, F. Sobott, and B. Brutschy, Rapid Commun. Mass Spectrom., 14, 859 (2000).
7) V. V. Laiko, N. I.Taranenko, V. D. Berkout, M. A. Yakshin, C. R. Prasad, H. Sang Lee, and V. M. Doroshenko, J. Am. Soc. Mass Spectrom., 13, 354 (2002).

8) P. Williams, Int. J. Mass Spectrom. Ion Processes, 131, 335 (1994).

9) S. Berkenkamp, M. Karas, and F. Hillenkamp, Proc. Natl. Acad. Sci. USA, 93, 7003 (1996).

10) K. Hiraoka, K. Murata, K. Aizawa, F. Matsushita, H. Fukasawa, and T. Sato, Rapid Commun. Mass Spectrom., 11, 474 (1997).

11) M. Karas, M. Glückmann, and J. Schafer, J. Mass Spectrom., 35, 1 (2000).

12) G. M. Hale and M. R. Querry, Appl. Opt., 12, 555 (1973).

Keywords: Laser spray, Electrospray, MALDI 